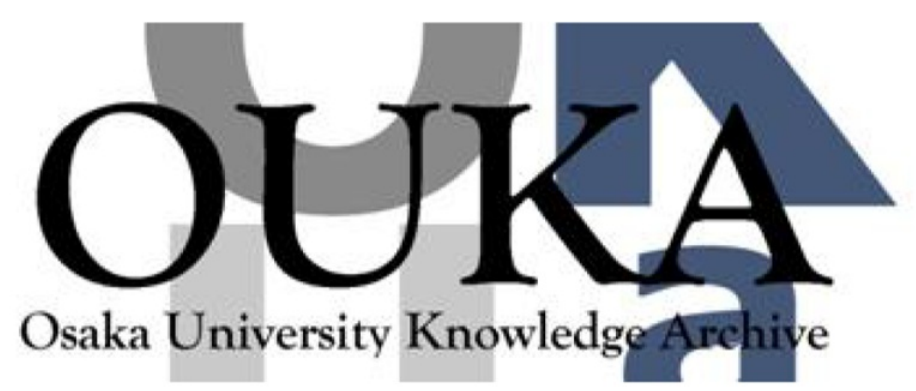

\begin{tabular}{|c|c|}
\hline Title & $\begin{array}{l}\text { Effect of Laser Intensity on Fast-Electron-Beam } \\
\text { Divergence in Solid-Density Plasmas }\end{array}$ \\
\hline Author (s) & $\begin{array}{l}\text { Green, J.S.; Ovchinnikov, V.M.; Evans, R.G. et } \\
\text { al. }\end{array}$ \\
\hline Citation & Physical Review Letters. 100(1) p.015003 \\
\hline Issue Date & $2008-01-11$ \\
\hline oaire:version & VoR \\
\hline URL & https://hdl. handle. net/11094/3437 \\
\hline rights & 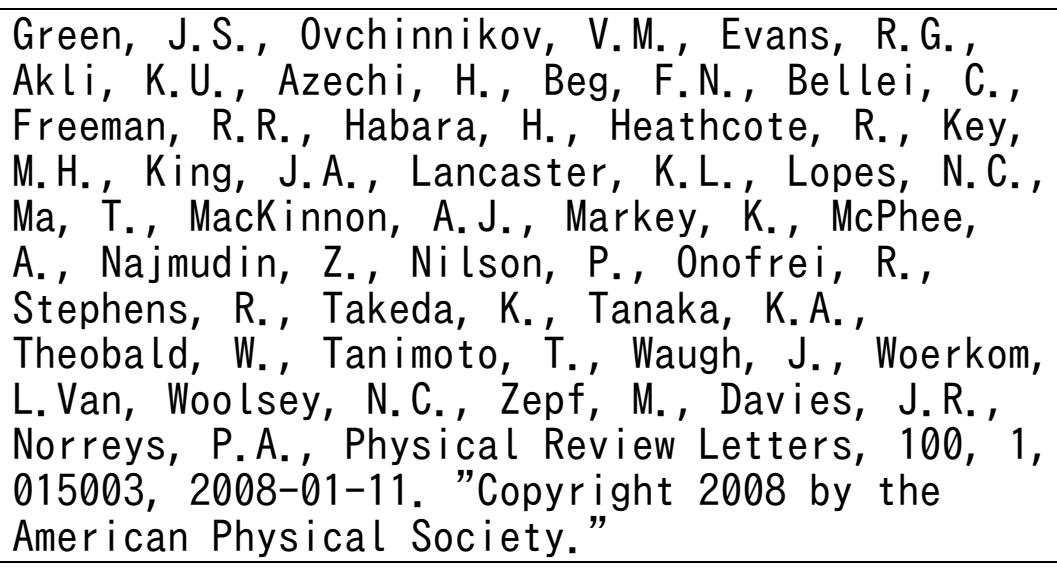 \\
\hline Note & \\
\hline
\end{tabular}

Osaka University Knowledge Archive : OUKA

https://ir. Library. osaka-u. ac. jp/ 


\title{
Effect of Laser Intensity on Fast-Electron-Beam Divergence in Solid-Density Plasmas
}

\author{
J. S. Green, ${ }^{1,2}$ V. M. Ovchinnikov, ${ }^{3}$ R. G. Evans,,${ }^{1,2}$ K. U. Akli, ${ }^{9}$ H. Azechi, ${ }^{8}$ F. N. Beg, ${ }^{6}$ C. Bellei, ${ }^{2}$ R. R. Freeman, ${ }^{3}$ \\ H. Habara, ${ }^{7}$ R. Heathcote, ${ }^{1}$ M. H. Key, ${ }^{4}$ J. A. King, ${ }^{6}$ K. L. Lancaster, ${ }^{1}$ N. C. Lopes, ${ }^{11}$ T. Ma, ${ }^{6}$ A. J. MacKinnon, ${ }^{4}$ \\ K. Markey, ${ }^{12}$ A. McPhee, ${ }^{4}$ Z. Najmudin, ${ }^{2}$ P. Nilson, ${ }^{10}$ R. Onofrei,${ }^{11}$ R. Stephens, ${ }^{9}$ K. Takeda, ${ }^{8}$ K. A. Tanaka, ${ }^{7}$ \\ W. Theobald, ${ }^{10}$ T. Tanimoto, ${ }^{7}$ J. Waugh, ${ }^{5}$ L. Van Woerkom, ${ }^{3}$ N. C. Woolsey, ${ }^{5}$ M. Zepf, ${ }^{12}$ J. R. Davies, ${ }^{11}$ and P. A. Norreys ${ }^{1,2}$ \\ ${ }^{1}$ Central Laser Facility, Rutherford Appleton Laboratory, Chilton, Oxon OX11 OQX, United Kingdom \\ ${ }^{2}$ Blackett Laboratory, Imperial College London, Prince Consort Road, London SW7 2BZ, United Kingdom \\ ${ }^{3}$ Department of Physics, The Ohio State University, Columbus, Ohio 43210-1117, USA \\ ${ }^{4}$ Lawrence Livermore National Laboratory, P.O. Box 808, Livermore, California 94550, USA \\ ${ }^{5}$ Department of Physics, University of York, Heslington, York YO10 5DD, United Kingdom \\ ${ }^{6}$ Department of Mechanical and Aerospace Engineering, University of California San Diego, \\ 9500 Gilman Drive 0411, La Jolla, California 92093-0411, USA \\ ${ }^{7}$ Graduate School of Engineering, Osaka University, Suita, 565-0871 Osaka, Japan \\ ${ }^{8}$ Institute of Laser Engineering, Osaka University, Suita, 565-0871 Osaka, Japan \\ ${ }^{9}$ General Atomics, P.O. Box 86508, San Diego, California 92186-5608, USA \\ ${ }^{10}$ Laboratory of Laser Energetics, University of Rochester, 250 East River Road, Rochester, New York 14623, USA \\ ${ }^{11}$ GoLP/Centro de Fisica dos Plasmas, Instituto Superior Tecnico, 1049-001 Lisbon, Portugal \\ ${ }^{12}$ School of Mathematics and Physics, Queen's University of Belfast, Belfast BT7 1NN, United Kingdom
}

(Received 14 May 2007; published 11 January 2008; corrected 15 January 2008)

\begin{abstract}
Metal foil targets were irradiated with $1 \mu \mathrm{m}$ wavelength $(\lambda)$ laser pulses of 5 ps duration and focused intensities $(I)$ of up to $4 \times 10^{19} \mathrm{~W} \mathrm{~cm}^{-2}$, giving values of both $I \lambda^{2}$ and pulse duration comparable to those required for fast ignition inertial fusion. The divergence of the electrons accelerated into the target was determined from spatially resolved measurements of x-ray $K_{\alpha}$ emission and from transverse probing of the plasma formed on the back of the foils. Comparison of the divergence with other published data shows that it increases with $I \lambda^{2}$ and is independent of pulse duration. Two-dimensional particle-in-cell simulations reproduce these results, indicating that it is a fundamental property of the laser-plasma interaction.
\end{abstract}

DOI: 10.1103/PhysRevLett.100.015003

PACS numbers: 52.38.Kd, 52.57.Kk

The fast ignition concept proposed in 1994 [1] has attracted extensive experimental and theoretical interest due to the reduced requirements for irradiation symmetry and drive energy compared with conventional inertial confinement fusion. In the fast ignitor scheme a separate laser pulse is used to produce a beam of electrons that deposit their energy inside the precompressed deuterium-tritium (DT) fuel pellet, heating a small hot spot fast enough that it does not expand hydrodynamically.

Atzeni [2] found that for a compressed core density of $400 \mathrm{~g} \mathrm{~cm}^{-3}$ at least $11 \mathrm{~kJ}$ must be deposited in a region with a radius of up to $15 \mu \mathrm{m}$ and a length of up to $30 \mu \mathrm{m}$ $\left(1.2 \mathrm{~g} \mathrm{~cm}^{-2}\right)$ in less than $16 \mathrm{ps}$. The electron energy required to give a stopping distance of $1.2 \mathrm{~g} \mathrm{~cm}^{-2}$ in $400 \mathrm{~g} \mathrm{~cm}^{-3}$ DT is $1.4 \mathrm{MeV}$ (varying weakly with density). Matching this energy to the ponderomotive potential gives an $I \lambda^{2}$ of $1.5 \times 10^{19} \mathrm{~W} \mathrm{~cm}^{-2} \mu \mathrm{m}^{2}$. This is lower than the values achieved by the current generation of high-intensity lasers, but on the other hand these machines use pulse durations much shorter than that required by fast ignition $(<1 \mathrm{ps})$.

Therefore, we decided to carry out an experimental campaign on the Vulcan PetaWatt laser using pulse durations of $5 \mathrm{ps}$, giving values of both $I \lambda^{2}$ and pulse duration comparable to those that would be required for an ignition laser, which are two key parameters in determining the physics of laser-plasma interactions. The spot radius and pulse energy were, however, much lower than those required for an ignition laser. Obtaining all of the required laser parameters simultaneously is clearly possible only with a laser far larger than any that currently exist. Here we report the results obtained on the divergence of the electrons accelerated into the target, which is a crucial parameter in determining the coupling of the electrons to the hot spot in fast ignition [3].

The experiment was performed with the Vulcan PetaWatt laser facility [4]. Vulcan PetaWatt is a Nd:glass laser that delivered intensities of up to $5 \times 10^{20} \mathrm{~W} \mathrm{~cm}^{-2}$ on target at an operating wavelength of $1.054 \mu \mathrm{m}$. Up to $300 \mathrm{~J}$ of laser energy was delivered on target. The laser was focused onto the target using an $f / 3$ parabola to a spot size in the range of 7-50 $\mu \mathrm{m}$ depending on the required focused intensity. The $p$-polarized laser radiation was incident on target at an angle of $40^{\circ}$.

The targets were $2 \mathrm{~mm} \times 2 \mathrm{~mm}$ Ti or $\mathrm{Cu}$ foils of 25-100 $\mu \mathrm{m}$ thickness and layered targets consisting of $25 \mu \mathrm{m} \mathrm{Cu}$ foils sandwiched between Al foils. Since the electron stopping due to electric field generation is more 
directly related to the distance traveled, rather than the target's areal density, it is reasonable to include these different target types in one combined data set. The main diagnostics employed to measure the electron beam divergence were $\mathrm{Cu}$ and $\mathrm{Ti} K_{\alpha}$ imagers and transverse optical probing, as these have been shown to provide complementary information [5]. The $\mathrm{Cu} K_{\alpha}$ imager, used to spatially resolve the $8.05 \mathrm{keV} \mathrm{Cu} K_{\alpha}$ emission from the target [6], consisted of a spherically bent Bragg crystal coupled to an image plate. The quartz $(21 \overline{3} 1)$ crystal focused the $8.05 \mathrm{keV} \mathrm{Cu} K_{\alpha}$ photons from the rear of the target onto the image plane where FujiFilm BAS-SR2040 image plate was placed. The Ti $K_{\alpha}$ imager consisted of a similar setup using a quartz (2023) crystal optimized to focus $4.5 \mathrm{keV} \mathrm{Ti}$ $K_{\alpha}$ emission.

A probe beam was created from a small part of the main beam that was frequency doubled to $2 \omega(527 \mathrm{~nm})$. The probe was timed to cross the target 200 ps after the main interaction pulse so as to image the plasma expansion on the front and rear surfaces. A $7-\mathrm{cm}$ diameter, $30-\mathrm{cm}$ focal length achromatic lens was used to collect the probe light after the target. The image was relayed outside of the target chamber to an eight-bit charge coupled device. The $f$ number of the imaging system was $f / 5$ and the magnification was $\times 8.5$. The spatial resolution was $5 \mu \mathrm{m}$. The maximum density probed was limited to roughly $5 \times$ $10^{19} \mathrm{~cm}^{-3}$ due to refraction in the steep density gradients.

Two sets of laser parameters were used to obtain data sets $A$ and $B$. Data set $A$ was obtained using a laser pulse duration of $5 \mathrm{ps}$. The target was displaced $180 \mu \mathrm{m}$ from best focus towards the parabolic mirror to provide a nominal defocused spot size of $50 \mu \mathrm{m}$. In practice, the spot was made up of a central $10 \mu \mathrm{m}$ full width half maximum (FWHM) spot, within which resided $20 \%$ of the energy, along with $\mathrm{a} \approx 50 \mu \mathrm{m}$ diameter lower intensity outer region. The focused laser intensity was calculated using the central high-intensity spot to be $1.5 \times 10^{19} \mathrm{~W} \mathrm{~cm}^{-2}$. Data set $B$ was obtained with the target placed at the optimum focus position. This provided a $7 \mu \mathrm{m}$ FWHM central peak and, with 5 ps pulse duration, an intensity on target of $4 \times 10^{19} \mathrm{~W} \mathrm{~cm}^{-2}$.

The $K_{\alpha}$ emission spot size was measured by taking a FWHM along the vertical axis of the target rear surface as the target and crystal were on the same horizontal plane. The $K_{\alpha}$ emission depth for each target was calculated using the method described by Lancaster et al. [5].

Figure 1 shows a plot of target depth vs $K_{\alpha}$ spot size for data set $A$. The electron beam divergence was inferred from the slope of the plotted radii vs target depth as $2 \times$ $\arctan (m)$, where $m$ is the slope. The straight line was fitted to the data using the least squares method weighted by the individual spot radii errors. These were governed primarily by fitting Gaussian profiles to the measured spot sizes, taking into account the measured signal-to-noise ratio. The divergence angle was calculated to be $29( \pm 7)^{\circ}$. The

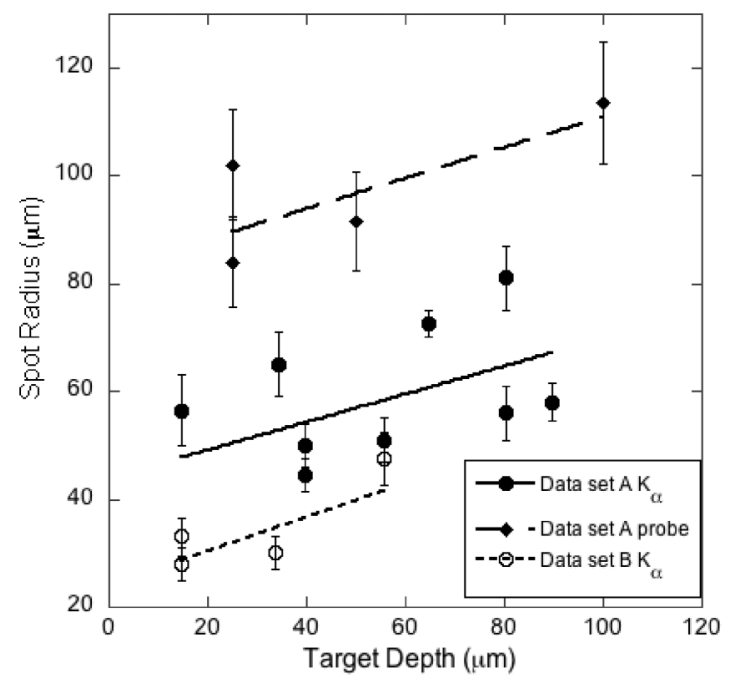

FIG. 1. $K_{\alpha}$ and shadowgraphy spot radii against target depth.

divergence angle was also inferred by measuring the FWHM of the plasma expansion on the rear surface of the targets from the shadowgrams for this data set. Fewer data points are included as most targets were too thick to exhibit a clear rear surface expansion. From the slope in Fig. 1 the divergence angle was measured as being $32( \pm 18)^{\circ}$. Data set $B$ is also plotted in Fig. 1. The divergence angle for this data set was seen to increase to $35( \pm 13)^{\circ}$.

A number of previous experiments with shorter pulse durations have obtained results on the divergence of the hot electrons. Early experiments using multiterawatt laser systems suggested a collimated fast electron flow in low $Z$ targets [7-9]. Simulations of these experiments indicated that the magnetic field formed at the edge of an electron beam inside the target resulted in beam collimation [10]. Later experiments used imaging of $K_{\alpha}$ emission from high $Z$ buried layer targets to measure electron beam divergence [11]. Results indicated a fast-electron beam with a $40^{\circ}$ spreading full angle. Santos et al. [12] used time-resolved optical diagnostics to measure the optical self-emission (generated either by incoherent transition or synchrotron radiation at the target rear surface) for different thickness targets and found a fast-electron-beam divergence angle of $34^{\circ}$. Kodama $K_{\alpha}[13,14]$ inferred a $20^{\circ}-30^{\circ}$ beam divergence using a similar technique at two different intensities. Most recently, Lancaster et al. [5] compared a range of imaging techniques to diagnose electron energy transport at the highest intensity range to date $\left(5 \times 10^{20} \mathrm{~W} \mathrm{~cm}^{-2}\right)$. Shadowgraphy and $K_{\alpha}$ imaging measurements revealed a larger divergence angle of more than $50^{\circ}$.

Figure 2 shows the divergence angle as a function of intensity on target for both newly obtained $K_{\alpha}$ data sets, as well as those in the published literature [5,11-14] described above. There is a clear trend of increasing beam divergence with intensity on target and that the beam divergence angle is most strongly influenced by this pa- 


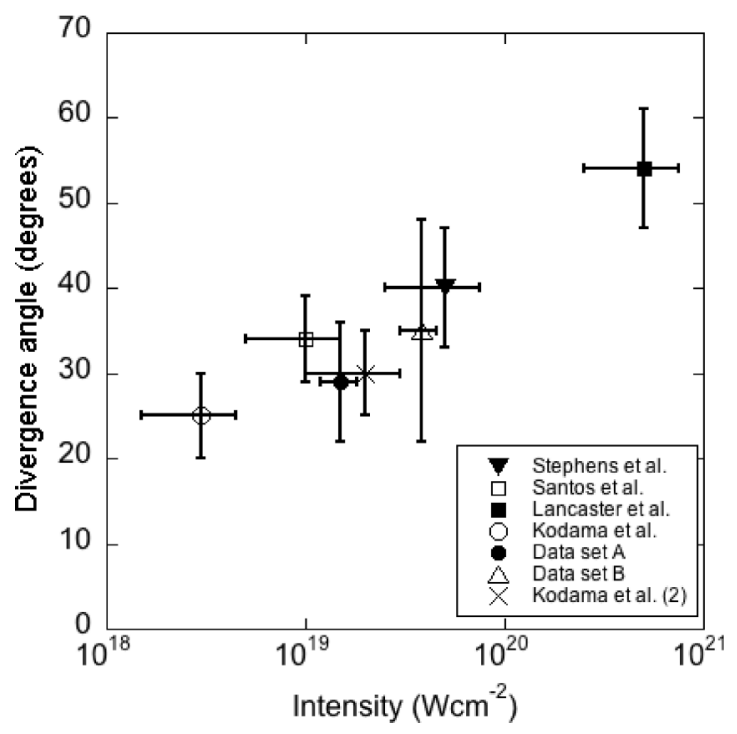

FIG. 2. Electron beam divergence as a function of intensity on target, along with other data published in the literature $[5,11-$ 14]. It is assumed that the errors in the other published work are similar, as the techniques employed are comparable.

rameter, particularly as those measurements were taken with very different focal spot conditions ranging from a circular focal spot of $7 \mu \mathrm{m}$ diameter [5] to an elongated spot of $30 \mu \mathrm{m} \times 40 \mu \mathrm{m}$ [14].

To test the validity of this conclusion, two-dimensional (2D) computer simulations were performed. The simulation of the whole process of the generation and transport of relativistic electrons by short, ultraintense laser pulses is an extremely difficult problem due to the large range of length scales that must be modeled. The process of electron acceleration in the laser focal spot is largely independent of the subsequent transport processes, although a rigorous treatment would have to allow for the fact that the large return current of "cold" electrons is an essential feature in maintaining the electron density in the interaction region. Any transport phenomena that hindered the return current, such as large magnetic fields or collision frequencies enhanced by strong plasma turbulence, would have a significant effect on the plasma environment in the acceleration region.

In the absence of fully self-consistent models for laser interactions with solid targets we have used the collisionless particle-in-cell (PIC) model OSIRIS [15] in 2D-3V geometry to model the data using as high a background density as is computationally feasible. The simulation grid covers $20 \mu \mathrm{m}$ (axially) by $40 \mu \mathrm{m}$ (transversely) with a 4000 by 8000 grid. There are 4 electrons and 4 ions per cell, equivalent to $1.6 \times 10^{5}$ particles per square wavelength. This enables the solid density to be set to $100 n_{c}$. The density rises linearly from zero to $100 n_{c}$ over $2.5 \mu \mathrm{m}$, using a two step profile $\left(0-30 n_{c}\right.$ in $2.5 \mu \mathrm{m}$ and $30-100 n_{c}$ in $1 \mu \mathrm{m})$. This increases the absorption of laser energy but does not markedly affect the temperature and divergence of the electrons. The laser rises linearly to its peak in $12 \mathrm{fs}$ and remains constant. The electron parameters are measured at $100 \mathrm{fs}$. All electrons with a Lorentz factor greater than 1.5 are binned to give the distribution in energy and angle. A plot of the fast electron angular distribution $n(\theta) d \theta$ for one simulation is shown in Fig. 3. The simulations use peak values of the vector potential of $a_{0}=2,5$, and 10 and spot sizes of 4,8 , and $16 \mu \mathrm{m}$.

The results are summarized in Table I, the temperature is the slope of the logarithmic energy distribution, and the angular distribution is measured to $50 \%$ of the forward peak. The spot size has rather little effect on temperature or divergence apart from slightly lower temperatures for the smallest spot size.

There are two possible explanations for the increase of divergence with intensity. Simulations since those of Wilks [16] have shown a rippling of the density contours at the target front surface on the scale of the laser wavelength in addition to the larger scale hole boring [17]. The beam divergence could be determined by the laser fields around the ripples, which in the simulations grow more quickly at higher irradiance. One might then expect the beam divergence with very short laser pulses, say less than $50 \mathrm{fs}$, to be smaller than for pulses of 100 fs or more. Simulations performed by Ren et al. [18] and Adam et al. [19] have suggested that the electron divergence is set by deflection in magnetic fields generated by the filamentation instability of the electron beam as it propagates through the target.

In the analysis by Silva et al. [20] for the filamentation instability for electron beams with water bag distributions, the threshold for the instability can be written $\theta^{2}=$ $n_{\text {hot }} / \gamma n_{\text {bg }}$ where $\theta$ is the divergence of the hot component, $n_{\text {hot }}$ and $n_{\mathrm{bg}}$ are the densities of the hot beam and background species, respectively, and $\gamma$ is the Lorentz factor for the beam electrons. In this analysis the divergence is

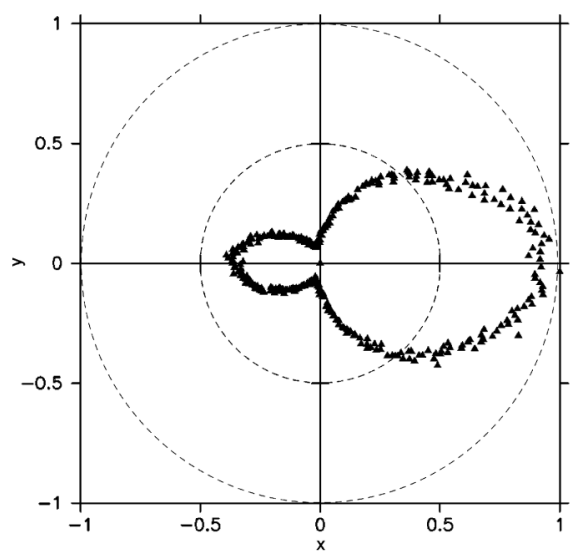

FIG. 3. Angular distribution $n(\theta) d \theta$ of the fast electrons in the plane of the simulation for an intensity of $10^{20} \mathrm{~W} \mathrm{~cm}^{-2}$ and spot diameter of $16 \mu \mathrm{m}$. $x$ is the direction of laser propagation and $y$ is the direction of the transverse electric field. $n(\theta) d \theta$ is normalized to 1 in the laser propagation direction. 
TABLE I. The slope temperature $(\mathrm{MeV}) /$ divergence half angle (degrees) for different intensities on target and focal spot diameter.

\begin{tabular}{ccrrr}
\hline \hline$a_{0}$ & Intensity & \multicolumn{1}{c}{$4 \mu \mathrm{m}$} & $8 \mu \mathrm{m}$ & $16 \mu \mathrm{m}$ \\
\hline 2 & $4 \times 10^{18} \mathrm{~W} \mathrm{~cm}^{-2}$ & $0.46 / 17$ & $0.61 / 19$ & $0.64 / 19$ \\
5 & $2 \times 10^{19} \mathrm{~W} \mathrm{~cm}^{-2}$ & $1.3 / 35$ & $1.8 / 35$ & $1.8 / 35$ \\
10 & $1 \times 10^{20} \mathrm{~W} \mathrm{~cm}^{-2}$ & $2.7 / 38$ & $3.4 / 47$ & $3.6 / 45$ \\
\hline \hline
\end{tabular}

approximately independent of intensity since $n_{\text {hot }}$ and $\gamma$ both increase approximately linearly with $a_{0}$ for relativistic laser intensities. However, the real situation with a large spread of beam energies is that there are many beam electrons with $\gamma \sim 1$ and a smaller number with large values of $\gamma$. Provided that the beam averaged $\gamma$ remains close to unity while $n_{\text {hot }}$ increases with intensity, then overall the beam divergence will increase with intensity as observed experimentally and in the simulations.

For the simulations with a background density of $100 n_{c}$ the divergence expected from this very simple model is around $8^{\circ}$ at $a_{0}=2$ and $17^{\circ}$ at $a_{0}=10$. This is about half of what is observed in the simulations, and some of the discrepancy may be due to differences between water bag and Maxwellian distributions for the transverse velocity components. In the situation of the experiments, it is difficult to know what value of background density to use in the presence of a largely unknown density gradient near the target surface.

The implications for fast ignition inertial fusion are significant. These results, together with the requirements detailed by Atzeni et al. [2], require the value of $I \lambda^{2}$ to be limited to $5 \times 10^{19} \mathrm{~W} \mathrm{~cm}^{-2} \mu \mathrm{m}^{2}$ for realistic ignition beam energies (i.e., $\leq 100 \mathrm{~kJ}$ ) [3]. Higher intensities on target will require control of the beam divergence pattern [21]. A beam divergence that increases with $I \lambda^{2}$ and that is independent of the spot size and profile has also been predicted to occur as a result of the decreasing collimating strength of the magnetic field generated inside the solid target $[22,23]$. This means that we cannot clearly distinguish between the effects of generation and transport in the experimental results. However, the OSIRIS results indicate that the increase in divergence with $I \lambda^{2}$ is not purely a transport effect, which means that this result is not limited to solid targets, and therefore can be extrapolated to fast ignition using cone guided geometries.

In conclusion, the fast-electron-beam divergence has been measured for intensity and pulse duration conditions of those required for fast ignition inertial fusion for the first time. The divergence has been measured to be
$29( \pm 7)^{\circ}$ and $32( \pm 18)^{\circ}$ by x-ray $K_{\alpha}$ imaging and optical shadowgraphy, respectively, at $1.5 \times 10^{19} \mathrm{~W} \mathrm{~cm}^{-2}$. A divergence of $35( \pm 13)^{\circ}$ was found at $4 \times 10^{19} \mathrm{~W} \mathrm{~cm}^{-2}$ by x-ray $K_{\alpha}$ imaging. An intensity dependence to the beam divergence has been revealed for the first time by comparing the new data with others published in the literature. The beam divergence appears to be independent of pulse duration. The intensity dependence on beam divergence is confirmed by 2D PIC simulations.

The authors gratefully acknowledge the assistance of the staff of the Central Laser Facility and the OSIRIS consortium [UCLA/IST(Portugal)/USC] for the use of OSIRIS. This work was supported by the U.K. Engineering and Physical Sciences Research Council and the Science and Technology Facilities Council. American colleagues acknowledge support from the U.S. Department of Energy Contract No. W-7405-Eng-48. Japanese colleagues acknowledge the Japan Society for the Promotion of Science.

[1] M. Tabak et al., Phys. Plasmas 1, 1626 (1994).

[2] S. Atzeni, Phys. Plasmas 6, 3316 (1999).

[3] J. J. Honrubia and J. Meyer-ter-Vehn, Nucl. Fusion 46, L25 (2006).

[4] C. N. Danson et al., Nucl. Fusion 44, S239 (2004).

[5] K. L. Lancaster et al., Phys. Rev. Lett. 98, 125002 (2007).

[6] D.C. Eder, G. Pretzler, E. Fill, K. Eidmann, and A. Saemann, Appl. Phys. B 70, 211 (2000).

[7] M. Tatarakis et al., Phys. Rev. Lett. 81, 999 (1998).

[8] M. Borghesi et al., Phys. Rev. Lett. 83, 4309 (1999).

[9] L. Gremillet et al., Phys. Rev. Lett. 83, 5015 (1999).

[10] J. R. Davies, A. R. Bell, and M. Tatarakis, Phys. Rev. E 59, 6032 (1999).

[11] R. B. Stephens et al., Phys. Rev. E 69, 066414 (2004).

[12] J. J. Santos et al., Phys. Rev. Lett. 89, 025001 (2002).

[13] R. Kodama et al., Nature (London) 412, 798 (2001).

[14] R. Kodama et al., Nucl. Fusion 44, S276 (2004).

[15] R. Hemker, Ph.D. dissertation, UCLA, 2000; R. A. Fonseca et al., Lect. Notes Comput. Sci. 2331, 342 (2002).

[16] S. C. Wilks, W.L. Kruer, M. Tabak, and A. B. Langdon, Phys. Rev. Lett. 69, 1383 (1992).

[17] A. Pukhov and J. Meyer-ter-Vehn, Phys. Rev. Lett. 79, 2686 (1997); B. F. Lasinski et al., Phys. Plasmas 6, 2041 (1999).

[18] C. Ren et al., Phys. Rev. Lett. 93, 185004 (2004).

[19] J. C. Adam, A. Heron, and G. Laval, Phys. Rev. Lett. 97, 205006 (2006).

[20] L. O. Silva et al., Phys. Plasmas 9, 2458 (2002).

[21] R. B. Campbell et al., Phys. Plasmas 10, 4169 (2003).

[22] J. R. Davies, Phys. Rev. E 68, 056404 (2003).

[23] J. R. Davies, J. S. Green, and P. A. Norreys, Plasma Phys. Controlled Fusion 48, 1181 (2006). 